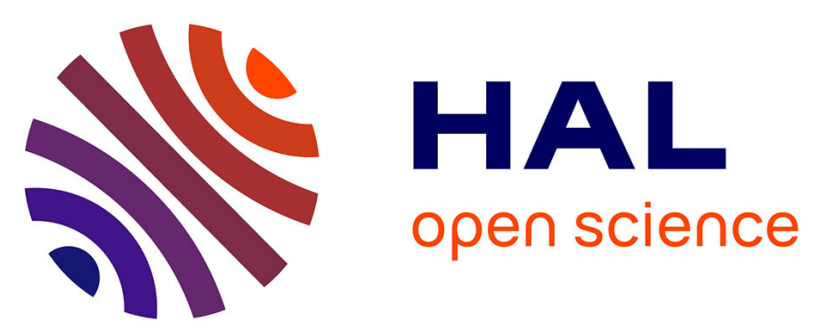

\title{
Enhanced single antenna interference cancellation from MMSE third-order complex Volterra filters
}

\author{
Mustapha Sadok, Jean-Pierre Delmas, Pascal Chevalier
}

\section{To cite this version:}

Mustapha Sadok, Jean-Pierre Delmas, Pascal Chevalier. Enhanced single antenna interference cancellation from MMSE third-order complex Volterra filters. ICASSP 2017 : 42nd International Conference on Acoustics, Speech and Signal Processing, Mar 2017, New Orleans, United States. pp.4197 - 4201, 10.1109/ICASSP.2017.7952947 . hal-01629454

\section{HAL Id: hal-01629454 \\ https://hal.science/hal-01629454}

Submitted on 6 Nov 2017

HAL is a multi-disciplinary open access archive for the deposit and dissemination of scientific research documents, whether they are published or not. The documents may come from teaching and research institutions in France or abroad, or from public or private research centers.
L'archive ouverte pluridisciplinaire HAL, est destinée au dépôt et à la diffusion de documents scientifiques de niveau recherche, publiés ou non, émanant des établissements d'enseignement et de recherche français ou étrangers, des laboratoires publics ou privés. 


\title{
ENHANCED SINGLE ANTENNA INTERFERENCE CANCELLATION FROM MMSE THIRD-ORDER COMPLEX VOLTERRA FILTERS
}

\author{
Mustapha Sadok $^{(1,2)}$, Jean Pierre Delmas ${ }^{(1)}$, and Pascal Chevalier ${ }^{(3,4)}$ \\ (1) Telecom SudParis, UMR CNRS 5157, Université Paris Saclay, 91011 Evry, France \\ (2) Institut National des Télécommunications et TIC, LaRATIC, Oran, Algeria \\ (3) CNAM, CEDRIC laboratory, 75003 Paris, France \\ (4) Thales-Communications-Security, HTE/AMS/TCP, 92622 Gennevilliers, France
}

\begin{abstract}
Widely linear (WL) filters have the capability to perform single antenna interference cancellation (SAIC) of one rectilinear or quasi-rectilinear co-channel interference (CCI). The SAIC technology for quasi-rectilinear signals is operational in GSM handsets but requires enhancements for both VAMOS standard, an evolution of GSM/EDGE standard, and FBMCOQAM networks, which are candidate for $5 \mathrm{G}$ mobile networks, in particular. For this reason, we propose in this paper a SAIC enhancement based on the use of third-order complex Volterra (CV) filtering, exploiting both the non-Gaussianity and the non-circularity of the signals up to the 6th-order. Limiting the analysis to rectilinear signals for space limitations, the performance of the proposed receiver are proved to outperform those of WL receivers for SAIC of one CCI.
\end{abstract}

Index Terms - Widely linear, SAIC, non-circular, nonGaussian, complex Volterra filter, CCI, MMSE.

\section{INTRODUCTION}

It is now well-known that one of the most important properties of WL filtering [1] is its capability to perform SAIC of one rectilinear (BPSK, ASK) or quasi-rectilinear (MSK, GMSK, OQAM) multi-user CCI, allowing the separation of two users from only one receive antenna [2-4]. The effectiveness of this concept jointly with its low complexity explain why it is operational in most of GSM handsets, allowing significant network's capacity gains for the GSM system $[4,5]$. However, as explained in [6-8], SAIC enhancements are required for new technologies such as voice services over adaptive multi-user channels on one slot (VAMOS) technology, an evolution of GSM standardized recently [9]. A similar need is also required to mitigate both inter-carrier interference (ICI) and CCI for networks which will use filter bank multi-carrier (FBMC) waveforms coupled with OQAM modulation, which are considered as promising candidates for the $5 \mathrm{G}$ mobile networks in particular [10].

In this context, the purpose of this paper is to propose and to analyze the performance of an enhanced SAIC receiver based on third-order CV filtering instead of WL filtering. While WL receivers exploit the second-order non-circularity of the signals, third-order $\mathrm{CV}$ receivers exploit both the nonGaussianity and the non-circularity of the signals up to the 6th-order [11], hence a performance gain. To introduce this new receiver, we adopt an MMSE approach and due to space limitations, we restrict the analysis to rectilinear signals and channels with no delay spread. Under these assumptions, it is shown in particular that the performance gain obtained in using the third-order $\mathrm{CV}$ receiver instead of the WL one depends on several parameters such as the constellation of the symbols and differential phases and delays between the signal of interest (SOI) and the CCI.

Note that the scarce papers dealing with $\mathrm{CV}$ filters for CCI mitigation correspond to [12-15]. While [12] deals with general $M$ th-order CV filters for detection and estimation in nonGaussian total noise with an application to CCI mitigation for $M=2$, [13-15] concern third-order CV filters for CCI mitigation. Nevertheless [13] is limited to CDMA communications networks, whereas [14] and [15] consider a beamforming application requiring at least two receive antennas.

\section{MODEL, STATISTICS AND PROBLEM FORMULATION}

\subsection{Signal model and statistics}

We consider a narrow-band antenna receiving the contribution of a rectilinear SOI corrupted by a rectilinear CCI and a background noise. Assuming propagation channels with no delay spread and perfect time and frequency synchronization of the SOI, the complex envelope of the signals at the output of the antenna can then be written as

$x(t)=\mu_{s} e^{i \phi_{s}} \sum_{k} a_{k} v(t-k T)+\mu_{j} e^{i \phi_{j}} \sum_{k} b_{k} v\left(t-k T-\tau_{j}\right)+n(t)$,

where $a_{k}$ and $b_{k}$ are real-valued zero-mean i.i.d. random variables corresponding to the symbols of the SOI and CCI respectively, independent to each other and with the zero-mean Gaussian background noise contribution $n(t) . T$ is the symbol period, $v(t)$ is the impulse response of the pulse shaping 
filter of both the SOI and CCI, $\mu_{s}$ and $\mu_{j}$ control the amplitude of the SOI and the CCI respectively, $\phi_{s}$ and $\phi_{j}$ are the phase of the SOI and CCI respectively and $\tau_{j} \in[0, T]$ is the delay of the CCI w.r.t. the SOI. Assuming that $v(t)$ is a raised cosine $1 / 2$ Nyquist filter and denoting by $x_{k}$ the sampled observation, at the symbol rate, at time $k T$ at the output of a matched filtering operation to the pulse shaping filter, we obtain:

$$
x_{k}=\mu_{s} e^{i \phi_{s}} a_{k} r(0)+\mu_{j} e^{i \phi_{j}} \sum_{\ell} b_{\ell} r\left((k-\ell) T-\tau_{j}\right)+n_{k},
$$

where $r(t) \stackrel{\text { def }}{=} v(t) \otimes v^{*}(-t)$ is the real-valued impulse response of a Nyquist filter where $\otimes$ is the convolution operation, whereas $n_{k}$ is the background noise contribution at the output of the matched filter, which is zero-mean, stationary, Gaussian and circular with power $\eta_{2}$.

We denote by $a_{k}^{\prime}$ and $b_{k}^{\prime}\left(\tau_{j}\right)$ the normalized symbol $a_{k}$ and quantity $\sum_{\ell} b_{\ell} r\left((k-\ell) T-\tau_{j}\right)$, respectively such that $\mathrm{E}\left(a_{k}^{\prime 2}\right)=\mathrm{E}\left(b_{k}^{\prime 2}\left(\tau_{j}\right)\right)=1$. Under these assumptions, (2) takes the form

$$
x_{k}=\sqrt{\pi_{s}} e^{i \phi_{s}} a_{k}^{\prime}+\sqrt{\pi_{j}} e^{i \phi_{j}} b_{k}^{\prime}\left(\tau_{j}\right)+n_{k},
$$

where $\pi_{s} \stackrel{\text { def }}{=} \mu_{s}^{2} \pi_{a} r^{2}(0)$ and $\pi_{j} \stackrel{\text { def }}{=} \mu_{j}^{2} \pi_{b}\left(\sum_{n} r^{2}\left((k-n) T-\tau_{j}\right)\right.$ (with $\pi_{a} \stackrel{\text { def }}{=} \mathrm{E}\left(a_{k}^{2}\right)$ and $\left.\pi_{b} \stackrel{\text { def }}{=} \mathrm{E}\left(b_{k}^{2}\right)\right)$ are the power of the SOI and CCI symbols, respectively. We will need in Section 4, the fourth and sixth-order moments of $b_{k}^{\prime}\left(\tau_{j}\right)$ given by

$$
\begin{aligned}
\kappa_{j} & \stackrel{\text { def }}{=} \mathrm{E}\left(b_{k}^{\prime 4}\left(\tau_{j}\right)\right)=\mathrm{E}\left(b_{k}^{4}\right) \sum_{\ell} r_{\ell}^{4}+6\left[\mathrm{E}\left(b_{k}^{2}\right)\right]^{2} \sum_{k<\ell} r_{k}^{2} r_{\ell}^{2} \\
\chi_{j} & \stackrel{\text { def }}{=} \mathrm{E}\left(b_{k}^{\prime 6}\left(\tau_{j}\right)\right)=\mathrm{E}\left(b_{k}^{6}\right) \sum_{\ell} r_{\ell}^{6}+15 \mathrm{E}\left(b_{k}^{2}\right) \mathrm{E}\left(b_{k}^{4}\right) \sum_{k \neq \ell} r_{k}^{4} r_{\ell}^{2} \\
& +90\left[\mathrm{E}\left(b_{k}^{2}\right)\right]^{3} \sum_{i<k<\ell} r_{i}^{2} r_{k}^{2} r_{\ell}^{2} .
\end{aligned}
$$

where $r_{k} \stackrel{\text { def }}{=} r\left(k T-\tau_{j}\right) / \sqrt{\pi_{b} \sum_{\ell} r^{2}\left(\ell T-\tau_{j}\right)}$.

\subsection{Problem formulation}

The problem addressed in this paper is to detect the symbols $a_{k}$ from the observations $x_{k}$ through an MMSE approach. Naturally, the best estimate $y_{k}$ of $a_{k}$ according to the MMSE criterion is the conditional expectation $y_{k}=\mathrm{E}\left(a_{k} \mid x_{k}\right)$. Note that for respectively circular or non-circular mutually Gaussian distributions of $\left(a_{k}, x_{k}\right)$, this conditional expectation becomes linear or widely linear [1] respectively. But for nonGaussian distribution of $\left(a_{k}, x_{k}\right)$, the derivation of this conditional expectation becomes generally non-linear in $x_{k}$ and needs this distribution, which is unknown in practice.

For this reason, we consider in this paper an approximation of this conditional expectation through the analysis of a particular class of non-linear filters corresponding to the $\mathrm{CV}$ filters, introduced for the first time in [12] and [16] in the context of detection and estimation. The general model of a memoryless full $M$ th-order time invariant $\mathrm{CV}$ filter is defined by

$$
y_{k}=\sum_{m=0}^{M} \sum_{q=0}^{m} w_{m, q}^{*} x_{k}^{m-q} x_{k}^{* q} .
$$

For $w_{0,0}=0$, rel. (6) defines a widely linear filter [1] for $M=1$ and a full complex linear-quadratic filter [17] for $M=2$. Rel. (6) can be compactly written in the form

$$
y_{k}=\widetilde{\mathbf{w}}^{H} \widetilde{\mathbf{x}}_{k},
$$

where $\widetilde{\mathbf{w}} \stackrel{\text { def }}{=}\left(w_{0,0}, w_{1,0}, w_{1,1}, w_{2,0}, w_{2,1}, w_{2,2}, \ldots, w_{M, M}\right)^{T}$ and $\widetilde{\mathbf{x}}_{k} \stackrel{\text { def }}{=}\left(1, x_{k}, x_{k}^{*}, x_{k}^{2}, x_{k} x_{k}^{*}, x_{k}^{* 2}, \ldots, x_{k}^{* M}\right)^{T}$ is the augmented observation. The problem of the optimal $M$ th-order $\mathrm{CV}$ filter is then to find $\widetilde{\mathbf{w}}$ minimizing the MSE between $y_{k}$ and $a_{k}$.

\section{THIRD-ORDER COMPLEX VOLTERRA MMSE RECEIVER}

\subsection{Mth-order complex Volterra MMSE filter}

The full $M$ th-order CV MMSE filter corresponds to the filter $\widetilde{\mathbf{w}}$ which minimizes the criterion $\operatorname{MSE}(\widetilde{\mathbf{w}})=\mathrm{E} \mid a_{k}-$ $\left.\widetilde{\mathbf{w}}^{H} \widetilde{\mathbf{x}}_{k}\right|^{2}$. This filter is classically given by

$$
\widetilde{\mathbf{w}}_{\mathrm{CV}-\mathrm{MMSE}}=\mathbf{R}_{\tilde{x}}^{-1} \mathbf{r}_{\tilde{x}, a},
$$

with $\mathbf{R}_{\tilde{x}} \stackrel{\text { def }}{=} \mathrm{E}\left(\widetilde{\mathbf{x}}_{k} \widetilde{\mathbf{x}}_{k}^{H}\right)$ and $\mathbf{r}_{\tilde{x}, a} \stackrel{\text { def }}{=} \mathrm{E}\left(\widetilde{\mathbf{x}}_{k} a_{k}^{*}\right)$. The MSE obtained with the full $M$ th-order CV MMSE filter (8) is given by

$$
\operatorname{MMSE} \stackrel{\text { def }}{=} \operatorname{MSE}\left[\widetilde{\mathbf{w}}_{\mathrm{CV}-\mathrm{MMSE}}\right]=\pi_{a}-\mathbf{r}_{\tilde{x}, a}^{H} \mathbf{R}_{\tilde{x}}^{-1} \mathbf{r}_{\tilde{x}, a} \text {. }
$$

If some components of the full $M$ th-order CV MMSE filter (6) are withdrawn, we obtain a partial $M$ th-order CV MMSE filter. The increase $\Delta_{C V}-$ MMSE of MMSE obtained by such a partial $M$ th-order $C V$ filter can be derived by partitioning $\widetilde{\mathbf{x}}_{k}$ into the retained, $\widetilde{\mathbf{x}}_{1, k}$, and the discarded, $\widetilde{\mathbf{x}}_{2, k}$, part. Applying the matrix inversion lemma to the partitioned augmented covariance matrix $\mathbf{R}_{\tilde{x}}$ written as

$$
\mathbf{R}_{\tilde{x}}=\left[\begin{array}{cc}
\mathbf{R}_{\tilde{x}_{11}} & \mathbf{R}_{\tilde{x}_{12}} \\
\mathbf{R}_{\tilde{x}_{12}}^{H} & \mathbf{R}_{\tilde{x}_{22}}
\end{array}\right],
$$

the increase of MMSE given by the partial $M$ th-order $\mathrm{CV}$ MMSE filter that only uses $\widetilde{\mathbf{x}}_{1, k}$ is given by

$$
\begin{gathered}
\Delta_{\mathrm{CV}-\mathrm{MMSE}}=\left(\mathbf{r}_{\tilde{x}_{2}, a}^{H}-\mathbf{r}_{\tilde{x}_{1}, a}^{H} \mathbf{R}_{\tilde{x}_{11}}^{-1} \mathbf{R}_{\tilde{x}_{12}}\right) \\
\left(\mathbf{R}_{\tilde{x}_{22}}-\mathbf{R}_{\tilde{x}_{12}}^{H} \mathbf{R}_{\tilde{x}_{11}}^{-1} \mathbf{R}_{\tilde{x}_{12}}\right)^{-1}\left(\mathbf{r}_{\tilde{x}_{2}, a}-\mathbf{R}_{\tilde{x}_{12}}^{H} \mathbf{R}_{\tilde{x}_{11}}^{-1} \mathbf{r}_{\tilde{x}_{1}, a}\right) \geq 0 .
\end{gathered}
$$

Consequently, the term $\widetilde{\mathbf{x}}_{2, k}$ does not bring any information $\left(\Delta_{\mathrm{CV}-\mathrm{MMSE}}=0\right)$ if it is not correlated with both $a_{k}$ and $\widetilde{\mathbf{x}}_{1, k}$. An example of such a situation, in the presence of zeromean signals with symmetric distributions, is the case where $\widetilde{\mathbf{x}}_{1, k}$ and $\widetilde{\mathbf{x}}_{2, k}$ gather the odd and even terms $m$ of (6), respectively. Consequently, only $M$ th-order CV MMSE filters such that $M$ is odd containing only polynomial terms of odd order $m$ ought to be used. For such filters, $\widetilde{\mathbf{w}}$ and $\widetilde{\mathbf{x}}_{k}$ are reduced to $\widetilde{\mathbf{w}}=\left(w_{1,0}, w_{1,1}, w_{3,0}, w_{3,1}, w_{3,2}, w_{3,3} \ldots, w_{M, M}\right)^{T}$ and 
$\widetilde{\mathbf{x}}_{k}=\left(x_{k}, x_{k}^{*}, x_{k}^{3}, x_{k}^{2} x_{k}^{*}, x_{k} x_{k}^{* 2}, x_{k}^{* 3} \ldots, x_{k}^{* M}\right)^{T}$. In this case, the components of $\widetilde{\mathbf{x}}_{k}$ can be rearranged in order as $\widetilde{\mathbf{x}}_{k}=$ $\left[\mathbf{x}_{k}^{\prime T}, \mathbf{x}_{k}^{\prime H}\right]^{T}$ where $\mathbf{x}_{k}^{\prime}=\left(x_{k}, x_{k}^{3}, x_{k}^{2} x_{k}^{*}, . ., x_{k}^{(M+1) / 2} x_{k}^{*(M-1) / 2}\right]^{T}$. Then, the partial $M$ th-order CV MMSE estimate $y_{k}$ of $a_{k}$ can be interpreted as the WL-MMSE estimate of $a_{k}$ given $\mathbf{x}_{k}^{\prime}$ and thus the partial $M$ th-order CV MMSE estimate inherits the properties of the WL-MMSE estimator [1]. In particular, for real-valued SOI symbols $a_{k}$, the estimate $y_{k}$ given by (7) is real-valued.

In contrast, if $\widetilde{\mathbf{x}}_{1, k}$ gathers the terms $\left(x_{k}, x_{k}^{*}\right)$ and $\widetilde{\mathbf{x}}_{2, k}$ the odd higher order terms, all the terms $\widetilde{\mathbf{x}}_{2, k}$ are correlated with $a_{k}$ and $\widetilde{\mathbf{x}}_{1, k}$ and thus contribute to decrease the MMSE with respect to that of the WL-MMSE filter. This proves the better performance, in terms of MMSE, of the partial $M$ th-order CV MMSE filter with only odd order terms with respect to the WL-MMSE filter.

Finally, note that in practice, $\mathbf{R}_{\tilde{x}}$ and $\mathbf{r}_{\tilde{x}, a}$ are not known a priori and have to be estimated from a training sequence correlated with the SOI symbols using a least square approach.

\subsection{Third-order complex Volterra MMSE filter}

We consider in this paper $M$ th-order CV MMSE filters with $M=3$ and odd order terms only (i.e. $m=1,3$ ), whose input/output relation is given by

$$
\begin{aligned}
y_{k} & =w_{1,0}^{*} x_{k}+w_{1,1}^{*} x_{k}^{*}+\overbrace{w_{3,0}^{*} x_{k}^{3}}^{0}+\overbrace{w_{3,1}^{*} x_{k}^{2} x_{k}^{*}}^{w_{3,2}^{*} x_{k} x_{k}^{2 *}}+\underbrace{w_{3,3}^{*} x_{k}^{3 *}}_{2} \stackrel{\text { def }}{=} \widetilde{\mathbf{w}}^{H} \widetilde{\mathbf{x}}_{k} . \\
& +\underbrace{}_{3}
\end{aligned}
$$

where $\widetilde{\mathbf{w}}$ is defined by (8), but where $\widetilde{\mathbf{w}}$ and $\widetilde{\mathbf{x}}_{k}$ are now restricted to $\tilde{\mathbf{w}}=\left[w_{1,0}, w_{1,1}, w_{3,0}, w_{3,1}, w_{3,2}, w_{3,3}\right]^{T}$ and $\widetilde{\mathbf{x}}_{k}=\left[x_{k}, x_{k}^{*}, x_{k}^{3}, x_{k}^{2} x_{k}^{*}, x_{k} x_{k}^{* 2}, x_{k}^{* 3}\right]$, respectively. A filter defined by (11) is called a full WL Cubic filter or a WL-C $(0$, 1, 2, 3) filter, i.e., a WL-Cubic filter taking into account the cubic terms 0, 1, 2 and 3. We will see in Section 4 that partial WL-Cubic MMSE filters with a single third-order term 0, 1, 2 or 3 in (11), called WL-C $(i)(i=0,1,2$ or 3$)$ allow us to obtain an MSE close to the MMSE (9) of the full WL-Cubic structure.

\subsection{Orthogonal decomposition}

To give an enlightening interpretation of a full or partial WLCubic MMSE filter allowing one to understand its better behavior w.r.t. to the WL-MMSE filter, we extend the interpretation of the latter introduced in [18] using the orthogonal projection theorem. To this aim, we note that all the terms of $\widetilde{\mathbf{x}}_{k}$ contain an SOI component through the orthogonal decomposition of $\widetilde{\mathbf{x}}_{k}$

$$
\widetilde{\mathbf{x}}_{k}=a_{k}\left(\frac{\mathbf{r}_{\tilde{x}, a}}{\pi_{a}}\right)+\widetilde{\mathbf{i}}_{k}
$$

From (12), the ratio of the powers of the SOI component and the associated global noise component at the output of an arbitrary WL-Cubic filter $\widetilde{\mathbf{w}}$, defines an SINR at its output:

$$
\operatorname{SINR}(\widetilde{\mathbf{w}})=\frac{\left|\widetilde{\mathbf{w}}^{H} \mathbf{r}_{\tilde{x}, a}\right|^{2}}{\pi_{a} \widetilde{\mathbf{w}}^{H} \mathbf{R}_{\tilde{i}} \widetilde{\mathbf{w}}},
$$

where $\mathbf{R}_{\tilde{i}} \stackrel{\text { def }}{=} \mathrm{E}\left(\widetilde{\mathbf{i}}_{k} \widetilde{\mathbf{i}}_{k}^{H}\right)$ is the covariance matrix of the component of $\widetilde{\mathbf{x}}_{k}$ (12) which gathers all its terms uncorrelated with the SOI symbol $a_{k}$. It is easy to prove that the WLCubic filters $\widetilde{\mathbf{w}}$ which maximize this SINR (13) are collinear to $\mathbf{R}_{\tilde{i}}^{-1} \mathbf{r}_{\tilde{x}, a}$. Applying the matrix inversion lemma to $\mathbf{R}_{\tilde{i}}=$ $\mathbf{R}_{\tilde{x}}-\pi_{a}^{-1} \mathbf{r}_{\tilde{x}, a} \mathbf{r}_{\tilde{x}, a}^{H}$ derived from the orthogonal decomposition (12), it is easy to verify that $\mathbf{R}_{\tilde{i}}^{-1} \mathbf{r}_{\tilde{x}, a}$ and $\mathbf{R}_{\tilde{x}}{ }^{-1} \mathbf{r}_{\tilde{x}, a}$ are collinear. Consequently the WL-Cubic filters $\widetilde{\mathbf{w}}$ which maximize the SINR (13) are collinear to $\widetilde{\mathbf{w}}_{\mathrm{CV}-\mathrm{MMSE}}$ (8) taking into account the new definitions of $\widetilde{\mathbf{w}}_{\mathrm{CV}-\mathrm{MMSE}}$ and $\widetilde{\mathbf{x}}_{k}$. The maximun of the SINR (13), denoted MSINR is thus given by:

$$
\operatorname{MSINR}=\frac{1}{\pi_{a}} \mathbf{r}_{\tilde{x}, a}^{H} \mathbf{R}_{\tilde{i}}^{-1} \mathbf{r}_{\tilde{x}, a}=\frac{\pi_{a}^{-1} \mathbf{r}_{\tilde{x}, a}^{H} \mathbf{R}_{\tilde{x}}^{-1} \mathbf{r}_{\tilde{x}, a}}{1-\pi_{a}^{-1} \mathbf{r}_{\tilde{x}, a}^{H} \mathbf{R}_{\tilde{x}}^{-1} \mathbf{r}_{\tilde{x}, a}}
$$

From (12) and (13), it is straightforward to deduce the following general relation linking the MSE and the SINR at the output $y_{k}$ of an arbitrary WL-Cubic filter $\widetilde{\mathbf{w}}$ :

$$
\operatorname{MSE}(\widetilde{\mathbf{w}})=\pi_{a}\left|1-\frac{\widetilde{\mathbf{w}}^{H} \mathbf{r}_{\tilde{x}, a}}{\pi_{a}}\right|^{2}+\frac{\left|\widetilde{\mathbf{w}}^{H} \mathbf{r}_{\tilde{x}, a}\right|^{2}}{\pi_{a} \operatorname{SINR}(\widetilde{\mathbf{w}})} .
$$

We deduce from (15) that the WL-Cubic filter $\widetilde{\mathbf{w}}$, which minimizes $\operatorname{MSE}(\widetilde{\mathbf{w}})$ under the constraint $\widetilde{\mathbf{w}}^{H} \mathbf{r}_{\tilde{x}, a}=\pi_{a}$ is also the WL-cubic filter which maximizes $\operatorname{SINR}(\widetilde{\mathbf{w}})$ under the same constraint. This shows that under the constraint $\widetilde{\mathbf{w}}^{H} \mathbf{r}_{\tilde{x}, a}=$ $\pi_{a}$, MSE minimization and SINR maximization are equivalent criteria, which gives a physical interpretation of the SINR criterion (13) in term of MSE minimization and we obtain

$$
\operatorname{MMSE}=\frac{\pi_{a}}{1+\operatorname{MSINR}} .
$$

Without this constraint $\widetilde{\mathbf{w}}^{H} \mathbf{r}_{\tilde{x}, a}=\pi_{a}$, (15) shows that the MSE minimization is no longer equivalent to SINR maximization, but $\widetilde{\mathbf{w}}_{\mathrm{CV}-\mathrm{MMSE}}$, which minimizes $\operatorname{MSE}(\widetilde{\mathbf{w}})$ also maximizes $\operatorname{SINR}(\widetilde{\mathbf{w}})$, but is not the only one.

\section{SINR AND BER PERFORMANCE ANALYSIS}

\subsection{SINR performance analysis}

For arbitrary rectilinear SOI and CCI signals, it is easy to derive the SINR at the output of the WL-MMSE receiver for strong CCI (i.e., for $\pi_{j} \gg \eta_{2}$ ) [3]. It is given by

$$
\operatorname{SINR}_{\mathrm{WL}} \approx \frac{2 \pi_{s}}{\eta_{2}}\left(1-\cos ^{2}(\Delta \phi)\right),
$$

where $\Delta \phi \stackrel{\text { def }}{=} \phi_{j}-\phi_{s}$. Rel. (17) shows that the WL-MMSE receiver performs the SAIC for $\Delta \phi \neq 0$, with decreasing performance as $\Delta \phi$ decreases to zero. In contrast, the derivation of the SINR at the output of the WL Cubic MMSE receiver is much more intricate. But using MATLAB symbolic algebra and calculus tools, we have proved that this output SINR follows the rational fraction form

$$
\mathrm{SINR}_{\mathrm{WL}-\mathrm{C}}=\frac{a_{P} \pi_{j}^{P}+\ldots+a_{1} \pi_{j}+a_{0}}{b_{P} \pi_{j}^{P}+. .+b_{1} \pi_{j}+b_{0}},
$$


where $P$ depends on the considered partial WL-Cubic MMSE structure and the coefficients $a_{0}, . ., a_{P}, b_{0}, . ., b_{P}$, are functions of $\pi_{s}, \eta_{2}, \kappa_{j}, \chi_{j}, \kappa_{s} \stackrel{\text { def }}{=} \mathrm{E}\left(a_{k}^{\prime}{ }^{4}\right), \chi_{s} \stackrel{\text { def }}{=} \mathrm{E}\left(a_{k}^{\prime 6}\right)$ and $\Delta \phi$.

In particular, the maximum value of $P$ is 4 for $\mathrm{WL}-\mathrm{C}(i)$ ( $i=0,1,2$ or 3 ) MMSE receivers. In this case, we have proved the following two asymptotic results according to the values of $\chi_{j}-\kappa_{j}^{2} \geq 0$ :

For $\chi_{j}-\kappa_{j}^{2}=0$ which occurs using (4) and (5) i.i.f. $\chi_{j}=$ $\kappa_{j}=1 \Leftrightarrow b_{k}^{\prime}\left(\tau_{j}\right)= \pm 1$, i.e., for synchronized BPSK CCI,

$$
\lim _{\pi_{j} \rightarrow \infty} \operatorname{SINR}_{\mathrm{WL}-\mathrm{C}(i)}=\frac{a_{3}}{b_{3}}=\frac{2 \pi_{s}}{\eta_{2}}, \forall \Delta \phi .
$$

For $\chi_{j}-\kappa_{j}^{2}>0$, i.e., for arbitrary rectilinear unsynchronized CCI:

$$
\lim _{\pi_{j} \rightarrow \infty} \operatorname{SINR}_{\mathrm{WL}-\mathrm{C}(i)}=\frac{a_{4}}{b_{4}}=\frac{2 \pi_{s}}{\eta_{2}}\left(1-\cos ^{2}(\Delta \phi)\right) .
$$

Rel. (19) shows that for synchronized BPSK sources, the CCI is completely removed whatever $\Delta \phi$ thanks to an amplitude discrimination between the sources. In this case, the performance gain with respect to the WL MMSE receiver increases with $\Delta \phi$. This amplitude discrimination is generally strong in full-duplex systems [19]. For non-synchronized or nonBPSK sources, despite the absence of gain brought by WL$\mathrm{C}(i) \mathrm{MMSE}$ receivers with respect to WL MMSE receivers for infinitely strong CCI, it is possible to show still significant gains in SINR for strong but not infinitely strong CCI. This is illustrated in Figs. 1 and 2 which show the SINR of the WLCubic(0) and WL receivers, as a function of $\Delta \phi$ for different values of $\tau_{j}$ for a roll-off of 0.3 , and as a function of $\tau_{j} / T$ for different values of the roll-off $\alpha$ for $\Delta \phi=0$, respectively. For both figures, the SOI and CCI have BPSK symbols with $\mathrm{SNR}=\pi_{s} / \eta_{2}=10 \mathrm{~dB}$ and INR $=\pi_{j} / \eta_{2}=30 \mathrm{~dB}$. These two figures show that this gain in SINR decreases when the roll off $\alpha$ of $v(t)$ decreases and $\tau_{j} \in[0, T / 2]$ increases. This is explained by the presence of increasing inter-symbol interference due to the pulse shaping filter, which Gaussianizes the CCI component $b_{k}^{\prime}\left(\tau_{j}\right)$ due to the central limit theorem and for which the gain decreases toward zero.

\subsection{BER performance analysis}

To complete the SINR performance analysis, let us present in this subsection the bit error rate (BER) obtained at the output of the WL-C(0) MMSE and WL MMSE filters, both using a simple sign detector for BPSK synchronized SOI and CCI. In Fig.3, this BER (blue curves) is compared to those of the MAP receiver which knows the parameters $\pi_{s}, \pi_{j}, \phi_{s}, \phi_{j}$ and $\eta_{2}$, as a function of the INR. The SINR at the outputs of the third-order WL-C(0) MMSE and WL MMSE filters are also plotted (red curves) in this figure, where $\mathrm{SNR}=10 \mathrm{~dB}$. This figure illustrates the power's discrimination allowed by the WL-C(0) MMSE receiver, for which its output SINR attains its minimum for SNR $\approx \mathrm{INR}$ and its maximum $2 \pi_{s} / \eta_{2}$ for strong CCI where the BER is minimum. So the BER and SINR obtained by the WL-C(0) MMSE receiver have consistent behaviors. This reinforces the meaning of the SINR defined by the orthogonal decomposition (13). We note that although the MAP receiver outperforms the WL-C(0) MMSE receiver, their BERs are very close for strong CCI.

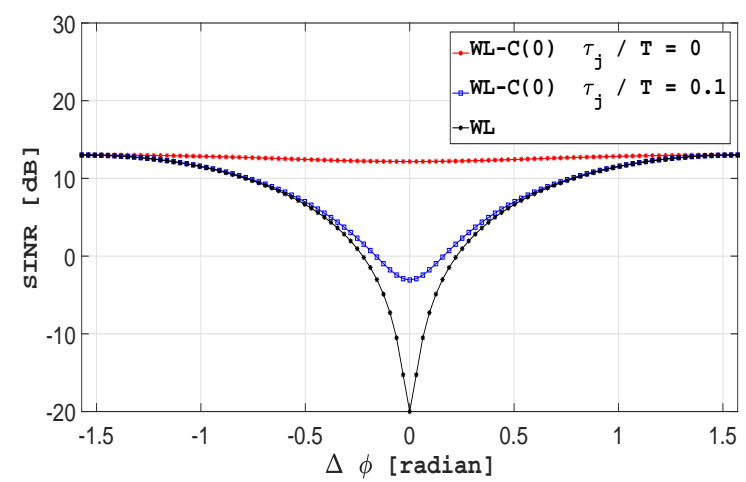

Fig.1 SINR $_{\mathrm{WL}-\mathrm{C}(0)}$ and $\mathrm{SINR}_{\mathrm{WL}}$ as a function of $\Delta \phi$ for $\alpha=0.3$.

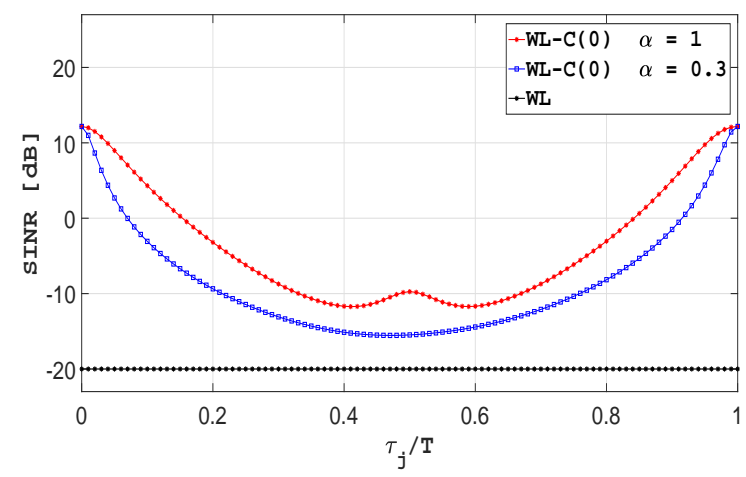

Fig.2 SINR $\operatorname{WL}_{\mathrm{C}(0)}$ and SINR ${ }_{\mathrm{WL}}$ as a function of $\tau_{j} / T$ for $\Delta \phi=0$.

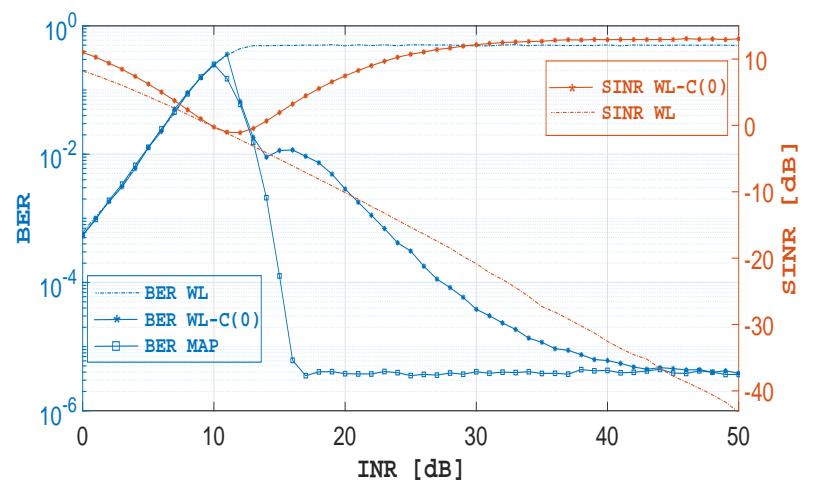

Fig.3 BER MAP $_{\text {BER }} \mathrm{BL}_{\mathrm{WL}(0)}, \mathrm{BER}_{\mathrm{WL}}, \mathrm{SINR}_{\mathrm{WL}-\mathrm{C}(0)}$ and $\mathrm{SINR}_{W L}$ as a function of INR for $\mathrm{SNR}=10 \mathrm{~dB}$.

\section{CONCLUSION}

An enhancement of the SAIC concept based on WL-Cubic MMSE receivers, has been proposed in this paper for rectilinear signals. This new receiver allows to obtain high performance gain with respect to WL-MMSE receiver depending on the symbols constellation, the pulse shaping filter and relative phase and delays between the SOI and the CCI. More detailed performance results will be presented elsewhere jointly with extensions of this receiver to second-order circular constellations. 


\section{REFERENCES}

[1] B. Picinbono and P. Chevalier, "Widely linear estimation with complex data", IEEE Trans. Signal Process., vol. 43, no. 8, pp. 2030-2033, Aug. 1995.

[2] H. Trigui and D.T.M. Slock, "Performance bounds for cochannel interference cancellation within the current GSM standard", Signal Proccessing, Elsevier, vol. 80, pp. 1335-1346, 2000.

[3] P. Chevalier and F. Pipon, "New Insights into optimal widely linear array receivers for the demodulation of BPSK, MSK and GMSK signals corrupted by noncircular interferences - Application to SAIC", IEEE Trans. Signal Process., vol. 54, no. 3, pp. 870-883, March 2006.

[4] R. Meyer, W.H. Gerstacker, R. Schober, and J.B. Huber, "A single antenna interference cancellation algorithm for increased GSM capacity", IEEE Trans. on Wireless Com., vol. 5, no. 7, pp. 1616-1621, July 2006.

[5] A. Mostafa, R. Kobylinski, I. Kostanic, and M. Austin, "Single antenna interference cancellation (SAIC) for GSM networks", Proc. VTC, vol. 2, pp. 1089-1093, Orlando, Florida, USA, Oct. 2004.

[6] M. Vutukuri, R. Malladi, K. Kuchi, and R. Koilpillai, "SAIC receiver algorithms for VAMOS downlink transmission", ISWCS, pp. 31-35, Aachen, Germany, Nov. 2011.

[7] D. Molteni and M. Nicoli, "Joint OSC receiver for evolved GSM/EDGE", IEEE Trans. Wireless Com., vol 12, no. 6, pp. 2608-2619, June 2013.

[8] M. Ruder, R. Meyer, F. Obernosterer, H. Kalveram, R. Schober, and W. Gerstacker, "Receiver concepts and resource allocation for OSC downlink transmission", IEEE Trans. Wireless Com., vol 13, no. 3, pp. 1568-1581, March 2014.

[9] R.C.D. Paiva, R.D. Vieira, R. Lida, F.M. Tavares, M. Saily, J. Hulkkonen, R. Jarvela, and K. Niemela, "GSM voice evolution using orthogonal subchannels", IEEE Commun. Magazine, pp. 80-86, Dec. 2012.

[10] G. Wunder et al. "5GNOW: Non-orthogonal asynchronous waveforms for future mobile applications", IEEE Commun. Magazine, pp. 97-105, Feb. 2014.

[11] B. Picinbono, "On Circularity", IEEE Trans. Signal Process., vol. 42, no. 12, pp. 3473-3482, Dec 1994.

[12] P. Chevalier, P. Duvaut, and B. Picinbono, "Le filtrage de Volterra transverse réel et complexe en traitement du signal", Traitement du Signal, Numéro spécial "non linéaire et non gaussien", vol 7, no. 5, pp 451-476, 1990.
[13] P. Comon, R. Liu, and D. Slock, "Path-wise wide-sense polynomial receiver for UMTS communications", 39th Conf. Com. Contr. Comput., Allerton, Illinois, Oct. 2001.

[14] A. Souloumiac, P. Chevalier, and C. Demeure, "Improvement in non-Gaussian jammers rejection with a non linear spatial filter", Proc ICASSP, Minneapolis (USA), pp. V670-V673, April 1993.

[15] P. Chevalier, A. Oukaci, and J.P. Delmas, "Third order widely nonlinear Volterra MVDR beamforming", Proc ICASSP, Prague, Czech Republic, May 2011.

[16] P. Chevalier, P. Duvaut, and B. Picinbono, "Complex transversal Volterra filters optimal for detection and estimation”, Proc ICASSP, Toronto, Canada, May 1991.

[17] P. Chevalier and B. Picinbono, "Complex linearquadratic systems for detection and array processing", IEEE Trans. Signal Process., vol 44, no. 10, pp. 26312634, Oct. 1996.

[18] P. Chevalier, J.P. Delmas, and A. Oukaci, "Properties, performance and practical interest of the widely linear MMSE beamformer for nonrectilinear signals", Signal Processing, vol. 97, pp. 269-281, April 2014.

[19] A. Ahmed and A.M. Eltawil, "All-digital selfinterference cancellation technique for full-duplex systems", IEEE Trans. Wireless Commun., vol. 14, no. 7, pp. 3519-3532, July 2015. 